\section{Case Reports in Nephrology and Dialysis}

Case Rep Nephrol Dial 2020;10:86-94

DOI: $10.1159 / 000509792$

Published online: July 30, 2020

(C) 2020 The Author(s)

Published by S. Karger AG, Basel www.karger.com/cnd

This article is licensed under the Creative Commons Attribution-NonCommercial 4.0 International License (CC BY-NC) (http://www.karger.com/Services/OpenAccessLicense). Usage and distribution for commercial purposes requires written permission.

\title{
Critical Coronavirus Disease 2019 in a Hemodialysis Patient: A Proposed Clinical Management Strategy
}

\author{
Charmaine Si Min Sia ${ }^{a}$ Samantha Hui Ling Cheong ${ }^{b}$ \\ Clara Lee Ying Ngoh ${ }^{a}$ Yi Hern Tan ${ }^{c}$ Weng Kin Wong ${ }^{a}$
}

aDivision of Nephrology, Department of Medicine, National University Hospital, Singapore, Singapore; 'bepartment of Medicine, National University Hospital, Singapore, Singapore; 'Department of Respiratory and Critical Care Medicine, Singapore General Hospital, Singapore, Singapore

\section{Keywords}

Coronavirus disease $2019 \cdot$ Infection control · Hemodialysis

\begin{abstract}
The coronavirus disease 2019 (COVID-19) pandemic has caused a catastrophic global health crisis. There is a lack of mitigation and clinical management strategies for COVID-19 in specific patient cohorts such as hemodialysis (HD) patients. We report our experience in treating the first case of COVID-19 in a HD patient in Singapore who had a severe clinical course including acute respiratory distress syndrome and propose a clinical management strategy. We propose a clinical workflow in managing such patients based on available evidence from literature review. We also highlight the importance of early recognition and intervention for disease control, dialysis support in an acute hospital isolation facility, deisolation protocol, and discharge planning due to prolonged viral shedding. The case highlights important points specific to a HD patient with a COVID-19 diagnosis, tailored interventions for each stage of the disease, and deisolation considerations in the recovery phase.




\section{Case Reports in Nephrology and Dialysis}

Sia et al.: COVID-19 Treatment Strategies for Hemodialysis Patients

\section{Introduction}

Since its outbreak, coronavirus disease 2019 (COVID-19) has claimed over 200,000 lives worldwide, disrupted livelihoods, and devastated the global economy. End-stage kidney disease (ESKD) patients are vulnerable due to intrinsic immunosuppression in addition to multiple comorbidities. There is a lack of clinical management strategies for COVID-19 in these patients. We report our experience in treating the first case of COVID-19 in a hemodialysis (HD) patient in Singapore and propose a clinical workflow. We found that early recognition and intervention are keys to disease mitigation and mortality reduction.

\section{Case Presentation}

A 45-year-old Singaporean Chinese female with ESKD secondary to presumed diabetic kidney disease on HD thrice a week via a right brachiocephalic arteriovenous fistula since January 2013 presented to the emergency department in March 2020 with a 2-day history of acute respiratory infection symptoms, fever, productive cough, rhinorrhea, and myalgia. She sought medical attention and was promptly admitted.

On admission, apart from four episodes of loose stools, she was clinically well without evidence of hemodynamic compromise. Her lungs were clear on auscultation and her chest X-ray (CXR) findings were unremarkable (Fig. 1a). Laboratory results revealed a total white cell count of $9.42 \times 10^{9} / \mathrm{L}$ with mild lymphopenia $0.54 \times 10^{9} / \mathrm{L}$, ferritin $1,225 \mu \mathrm{g} / \mathrm{L}$, and lactate dehydrogenase (LDH) $684 \mathrm{U} / \mathrm{L}$. Nasopharyngeal swabs were performed using the locally developed Fortitude Kit 2.0 (Agency for Science, Technology and Research, Singapore), which runs on a reverse transcription polymerase chain reaction (PCR) method of detecting SARSCoV-2 genetic material. Due to high clinical suspicion for COVID-19, when her first nasopharyngeal swab returned negative, further sputum, serum, and stool PCR samples were obtained while awaiting repeat nasopharyngeal swab results. All further samples subsequently returned positive. Her other comorbidities included well-controlled type 1 diabetes mellitus, hypertension, dyslipidemia, and ischemic heart disease. Of note, her mother who resided in the same household had developed fever 5 days prior and was later diagnosed with COVID19.

\section{Clinical Course}

Management of Acute Respiratory Distress Syndrome

On day 4 of admission, she developed respiratory distress and hypoxemia requiring $2 \mathrm{~L}$ of oxygen via nasal prongs. A repeat CXR revealed new left upper zone consolidation (Fig. 1b). She was transferred to the medical intensive care unit (ICU) for further monitoring. She continued to require low-flow oxygen supplementation to maintain her saturations. As her condition remained stable, she was transferred back to the general ward on day 6 . The next day, she rapidly deteriorated into severe acute respiratory distress syndrome. She was intubated and initiated on protective low-flow tidal volume mechanical lung ventilation and vasopressor support. This time, her CXR revealed widespread bilateral infiltrates (Fig. 1c). As she had high ventilatory requirements with persistent hypoxia despite $100 \%$ fraction of inspired oxygen $\left(\mathrm{FiO}_{2}\right)$ as well as positive end-expiratory pressure of $18 \mathrm{~cm} \mathrm{H}_{2} \mathrm{O}$ on assist control ventilation, a decision for early neuromuscular blockade and prone positioning was made. Despite this, 


\section{Case Reports in Nephrology and Dialysis}

Sia et al.: COVID-19 Treatment Strategies for Hemodialysis Patients

she remained in hypercapnic failure, meeting the indications for extracorporeal membrane oxygenation. However, she was deemed unsuitable due to underlying venous access issues and was continued on supportive therapy. She had a history of central vein stenosis which required stenting of the right subclavian vein and superior vena cava. On her most recent computed tomography (CT) central venogram done in September 2019, there had already been a 50-70\% luminal in-stent stenosis. Additionally, she also had chronic right internal jugular vein occlusion. She started to show clinical improvement on day 9, was extubated on day 11, and was transferred to the general ward on day 13 (Fig. 2).

\section{Dialysis Support}

While in the general ward, she was clinically euvolemic and underwent conventional dialysis to her dry weight of $60 \mathrm{~kg}$. While in the medical ICU, she was converted to continuous venovenous hemodiafiltration (CVVHDF) with regional citrate anticoagulation and a calculated effluent dose of $30 \mathrm{~mL} / \mathrm{kg} / \mathrm{h}$ for $55 \mathrm{~h}$ with one episode of filter clotting at $4 \mathrm{~h}$ in between. She was kept on even balance for the first $24 \mathrm{~h}$ and subsequently placed on a net fluid removal rate of $50 \mathrm{~mL} / \mathrm{h}$ to optimize ventilation once her hemodynamics had stabilized. Intermittent dialysis was resumed after extubation and weaning of vasopressor support.

\section{Recovery}

The patient was deisolated after two negative nasopharyngeal PCR test taken $24 \mathrm{~h}$ apart had been obtained on days 28 and 29. Notably, her nasopharyngeal PCR test returned negative on day 21 but turned positive again on day 22 .

\section{Treatment Agents}

The patient was persistently febrile until day 10 and a trial of therapeutics was administered given rapid deterioration of clinical status. This included a single dose of oral Kaletra ${ }^{\circledR}$ (lopinavir $200 \mathrm{mg}$, ritonavir $50 \mathrm{mg}$ ), oral hydroxychloroquine (400 mg daily for 2 days then $200 \mathrm{mg}$ twice a day for 6 days), and subcutaneous beta-interferon 1B ( $250 \mu \mathrm{g}$ daily) for 5 days. Therapeutics were stopped early in view of clinical improvement.

\section{Discussion}

Our experience in managing this case highlights several learning points in diagnosis and management.

\section{Diagnosis and Clinical Manifestations}

The patient's initial normal CXR was falsely reassuring and inadequate to differentiate between mild and severe-type COVID-19 disease. In February 2020, the Chinese Society of Radiology published guidelines suggesting that CT imaging improves the accuracy of COVID19 diagnosis and that further CT image visuals aid in the quantification of disease severity [1]. However, this is not widely adopted in local practice due to resource constraints and infectious control reasons.

It is unclear why her first nasopharyngeal PCR test returned negative. We postulate that viral shedding may not have reached the PCR kit detection limit or that the initial swab was inadequately performed. Nonetheless, the locally developed kits have been validated in Singapore and manufactured according to regulations. The analytical limit of detection of the Fortitude Kit is 25 copies per reaction [2]. Alternatively, this could have been a false-negative 


\section{Case Reports in Nephrology and Dialysis}

Case Rep Nephrol Dial 2020;10:86-94

DOI: 10.1159/000509792

(c) 2020 The Author(s). Published by S. Karger AG, Basel www.karger.com/cnd

Sia et al.: COVID-19 Treatment Strategies for Hemodialysis Patients

result; as the incidence of COVID-19 increases, the negative predictive value of the test kit decreases, but exact epidemiological data for this are lacking.

There are controversies regarding the clinical course of COVID-19 in ESKD patients. A case series of an outbreak in a dialysis center in Wuhan, where HD patients were observed to have a milder disease course or total absence of symptoms with lower levels of serum inflammatory cytokines, suggested that this is due to the inability to mount an effective cellular immune response and hence an absence of cytokine release syndrome [3]. However, our patient had a rapid and fulminant disease course. So far, there are no specific studies using biomarkers to predict the risk of deterioration among COVID-19 ESKD patients, although ferritin and LDH have been found in many studies to be associated with severe illness and mortality in COVID-19 [4,5]. Our patient did have high LDH and ferritin on admission. A study to address the correlation between biomarkers and disease severity in ESKD patients is urgently warranted.

The symptoms of COVID-19 are highly variable and, as with all viruses, self-limiting [6]. The major clinical patterns include mild acute respiratory infection, nonfatal pneumonia, and acute respiratory distress syndrome. Infrequently, gastroenteritis may precede respiratory symptoms [6]. While a large majority of cases develop mild disease and recover, the individual patient trajectory tends to wax and wane, and progression to critical disease is unpredictable, as with this case [6]. Our patient had a biphasic clinical evolution where she had mild symptoms that transiently improved before deterioration into critical disease. This clinical pattern has also been described in other studies and in some cases, despite conversion to PCR negativity [7]. This is hypothesized to be a two-phase immune response with initial adaptive immunity to eliminate the virus, resulting in mild symptomology and a subsequent cytokine release syndrome with persistent viral infection leading to critical disease [7].

Risk factors for severe COVID-19 illness include older age and chronic conditions, especially chronic kidney disease as well as respiratory and cardiovascular disease [8]. The estimated mortality rate of 3-6\% in COVID-19 is lower compared to Middle East respiratory syndrome coronavirus (MERS-CoV) (35\%) and severe acute respiratory syndrome coronavirus (2003 SARS-CoV) (10\%) [9]. In contrast, the mortality rate in HD patients with COVID-19 is up to $30 \%$ as reported in the Italian and Spanish cohorts $[10,11]$.

Prolonged viral shedding is not unexpected in these patients as this has also been observed in other immunocompromised patients with COVID-19. During the previous 2003 SARS-CoV outbreak, dialysis patients were noted to have persistent viral shedding of up to 8 weeks [12]. Arguably, a positive viral PCR test does not equate the presence of live viruses and therefore may not translate to infectivity. However, there is no robust method to prove this for COVID-19 disease, and those in convalescence may still be infectious. Given the highly contagious nature of this disease, we keeping PCR-positive patients isolated in an effort to contain the pandemic. This will result in prolonged hospitalizations and consequent strain on healthcare resources. Nonetheless, caution must be exercised when deisolating ESKD patients to mitigate disease spread at outpatient dialysis centers, given the possibility of re-emergence of viral shedding after an initial period of negativity, as with this case.

A few speculations can be made about the fluctuant PCR results. We postulate that the sampling method could have been inadequate and highlight the importance of repeat sampling to ensure accuracy of a true-negative test. Perhaps a combination of PCR samples from different sites could also be obtained to maximize sensitivity; however, this should be weighed against resource management. Secondly, this could reflect reinfection. During the 2003 SARS$\mathrm{CoV}$ outbreak, Lin et al. [13] found that neutralizing antibodies are acquired late after recov- 
ery. However, our patient showed continued clinical improvement with resolution of symptoms, hence reinfection is unlikely.

\section{Clinical Management}

Nonpharmaceutical interventions remain pivotal as there are still no licensed therapeutics, and current agents are administered based on in vitro and observational studies. Although it is unclear whether the therapeutics administered benefited our patient significantly, they did not result in harm. As we await definite results from ongoing trials, it is reasonable to administer a treatment regimen based on current observations of efficacy. Given the potential for rapid deterioration, early recognition, intervention, and escalation of care to a controlled ICU setting where adjunctive measures are readily available are paramount to avoid delays in treatment. There is a need to further streamline protocols to facilitate effective resource planning and mitigation strategies in the face of overwhelming demands. We examined international guidelines provided by different centers [14] and proposed a workflow based on the time points of the clinical course of COVID-19 disease in ESKD patients (Fig. 3). We define critical COVID-19 disease as severe respiratory infection requiring mechanical ventilation.

\section{Pre-Critical Phase}

During this nonsevere symptomatic phase, the focus lies mainly on infection control and early recognition of clinical deterioration with escalation of care. COVID-19-positive patients should undergo dialysis in an isolation facility with separate dialysis machines. Medical personnel attending to the patient should don full personal protective equipment. As ESKD patients have lower reserves alongside multiple comorbidities, deterioration if imminent will be rapid. Hence, we recommend management in an inpatient setting where escalation of care can be facilitated. Additionally, we recommend to aim towards euvolemia or a slight volume contracted state during conventional HD to avoid confounding respiratory symptoms. Bearing in mind imminent resource scarcity and medical staff fatigue, patients should be cohorted in the same area in a bid to minimize unnecessary exposure for noninfected persons and to maximize effective resource use such as re-usage of personal protective equipment components or cohorting of dialysis machines.

\section{Critical Phase}

In our institution, we advocate a model of early intensive care and mechanical ventilation. Patients with COVID-19 pneumonia requiring any level of supplemental oxygen to maintain saturations $>92 \%$ are nursed in the ICU. Orotracheal intubation is recommended at $\mathrm{FiO}_{2}$ requirements of $\geq 0.4$. The subsequent acute respiratory distress syndrome management is otherwise as per standard guidelines.

We recommend simplification of dialysis prescriptions to minimize nursing exposure to the patient. For continuous renal replacement therapy, the key principles are to maximize filter life and reduce dialysate expenditure. Continuous venovenous HD or predilution continuous venovenous hemofiltration/CVVHDF should be instituted to minimize filtration fraction. This is to be carried out at the minimum required effluent dose. In our case, we faced issues with filter clotting on CVVHDF which resulted in additional nursing exposure to the patient. Alternatively, prolonged intermittent renal replacement therapy can be considered to free dialysis machines for use. Anticoagulation should be used unless otherwise contraindicated. We recommend restrictive fluid management as soon as hemodynamics allow to optimize venti- 


\section{Case Reports in Nephrology and Dialysis}

Sia et al.: COVID-19 Treatment Strategies for Hemodialysis Patients

lation, facilitate extubation, and reduce the need for additional intermittent dialysis sessions as the patient's condition improves.

Attention should be directed towards preserving vascular access sites and resources for these patients as well. We advocate for catheter exchange or early conversion to a tunneled catheter when there is catheter dysfunction rather than reinsertion at multiple sites as far as the clinical condition allows. Right internal jugular access is preferred to avoid re-siting in the event that prone positioning has to be undertaken.

\section{Recovery Phase}

During this phase, infection control and mitigation strategies are paramount. Patients should remain segregated until there is consistent absence of viral shedding. We recommend deisolation only when there is sustained defervescence for $\geq 72 \mathrm{~h}$, resolution of respiratory symptoms for $\geq 72 \mathrm{~h}$ (for example the absence of cough or shortness of breath), at least 10 days have passed since symptoms first arose, and at least two consecutively negative nasopharyngeal PCR test taken $24 \mathrm{~h}$ apart. This is in reference to the Centers for Disease Control and Prevention guidelines [15]. Until then, dialysis should be continued in an isolation facility. As viral shedding is expected to be prolonged, a strategy to conserve hospital resources in these otherwise clinically well patients includes stepdown care to a community isolation facility. Such patients should also be cohorted at a specific community dialysis center with personal transportation using a fixed route.

\section{Conclusions}

Our patient developed severe manifestations of COVID-19 but made a full recovery with supportive care. Due to prolonged viral shedding, she had a month-long hospitalization. With the protracted pandemic, the number of affected HD patients is only expected to rise, with potential outbreaks within dialysis centers, placing a threat to healthcare resources and dialysis logistics; this is already imminent in other larger countries where critical care facilities fall short of demand, resulting in suboptimal care. Center-specific protocols should be adopted to facilitate effective resource planning and mitigation strategies in the face of overwhelming demands.

\section{Statement of Ethics}

The patient gave written informed consent for publication of her case including publication of images. This case study was conducted ethically in accordance with the World Medical Association Declaration of Helsinki.

\section{Conflict of Interest Statement}

The authors have no conflicts of interest to declare. 


\section{Case Reports in Nephrology and Dialysis}

\section{Funding Sources}

The authors received no specific funding for this work.

\section{Author Contributions}

C.S.M. Sia, Y.H. Tan, and W.K. Wong contributed to patient care. C.S.M. Sia and S.H.L. Cheong contributed to the writing of the manuscript. Y.H. Tan, C.L.Y. Ngoh, and W.K. Wong contributed to the editing of the manuscript.

\section{References}

1 Chinese Society of Radiology. Radiological diagnosis of new coronavirus infected pneumonitis: expert recommendation from the Chinese Society of Radiology (first edition). Chin J Radiol. 2020;54:E001-E.

2 Agency for Science, Technology and Research, Singapore. From Singapore to the world: where Fortitude Kit has been deployed globally. Updated 2020 March 27. Available from: www.a-star.edu.sg/news-and-events/ a-star-news/news/covid-19/from-singapore-to-the-world-where-fortitude-kit-2.0-has-been-deployedglobally [cited April 20, 2020].

3 Ma Y, Diao B, Lv X, Zhu J, Liang W, Liu L, et al. 2019 novel coronavirus disease in hemodialysis (HD) patients: report from one HD center in Wuhan, China. medRxiv. doi: 10.1101/2020.02.24.20027201 [Epub ahead of print].

4 Henry BM, de Oliveira MHS, Benoit S, Plebani M, Lippi G. Hematologic, biochemical and immune biomarker abnormalities associated with severe illness and mortality in coronavirus disease 2019 (COVID-19): a metaanalysis. Clin Chem Lab Med. 2020 Jun;58(7):1021-8.

5 Li X, Xu S, Yu M, Wang K, Tao Y, Zhou Y, et al. Risk factors for severity and mortality in adult COVID-19 inpatients in Wuhan. J Allergy Clin Immunol. 2020 Jul;146(1):110-8.

6 Guan WJ, Ni ZY, Hu Y, Liang WH, Ou CQ, He JX, et al.; China Medical Treatment Expert Group for Covid-19. Clinical Characteristics of Coronavirus Disease 2019 in China. N Engl J Med. 2020 Apr;382(18):1708-20.

7 Shi Y, Wang Y, Shao C, Huang J, Gan J, Huang X, et al. COVID-19 infection: the perspectives on immune responses. Cell Death Differ. 2020 May;27(5):1451-4.

8 Bhatraju PK, Ghassemieh BJ, Nichols M, Kim R, Jerome KR, Nalla AK, et al. Covid-19 in critically ill patients in the Seattle region - case series. N Engl J Med. 2020 May;382(21):2012-22.

9 Fauci AS, Lane HC, Redfield RR. Covid-19 - navigating the uncharted. N Engl J Med. 2020 Mar;382(13):12689.

10 Goicoechea M, Sánchez Cámara LA, Macías N, Muñoz de Morales A, Rojas ÁG, Bascuñana A, et al. COVID-19: clinical course and outcomes of 36 hemodialysis patients in Spain. Kidney Int. 2020 Jul;98(1):27-34.

11 Alberici F, Delbarba E, Manenti C, Econimo L, Valerio F, Pola A, et al. A report from the Brescia Renal COVID Task Force on the clinical characteristics and short-term outcome of hemodialysis patients with SARS-CoV-2 infection. Kidney Int. 2020 Jul;98(1):20-6.

12 Kwan BC, Leung CB, Szeto CC, Wong VW, Cheng YL, Yu AW, et al. Severe acute respiratory syndrome in dialysis patients. J Am Soc Nephrol. 2004 Jul;15(7):1883-8.

13 Lin Q, Zhu L, Ni Z, Meng H, You L. Duration of serum neutralizing antibodies for SARS-CoV-2: Lessons from SARS-CoV infection. J Microbiol Immunol Infect. doi: 10.1016/j.jmii.2020.03.015 [Epub ahead of print].

14 Alberici F, Delbarba E, Manenti C, Econimo L, Valerio F, Pola A, et al.; Brescia Renal COVID Task Force. Management Of Patients On Dialysis And With Kidney Transplant During SARS-COV-2 (COVID-19) Pandemic in Brescia, Italy. Kidney Int Rep. 2020 Apr;5(5):580-5.

15 Centers for Disease Control and Prevention. Discontinuation of transmission-based precautions and disposition of patients with COVID-19 in healthcare settings (interim guidance). Updated May 2, 2020. Available from: www.cdc.gov/coronavirus/2019-ncov/hcp/disposition-hospitalized-patients.html [cited June 5, 2020]. 


\section{Case Reports in Nephrology and Dialysis}
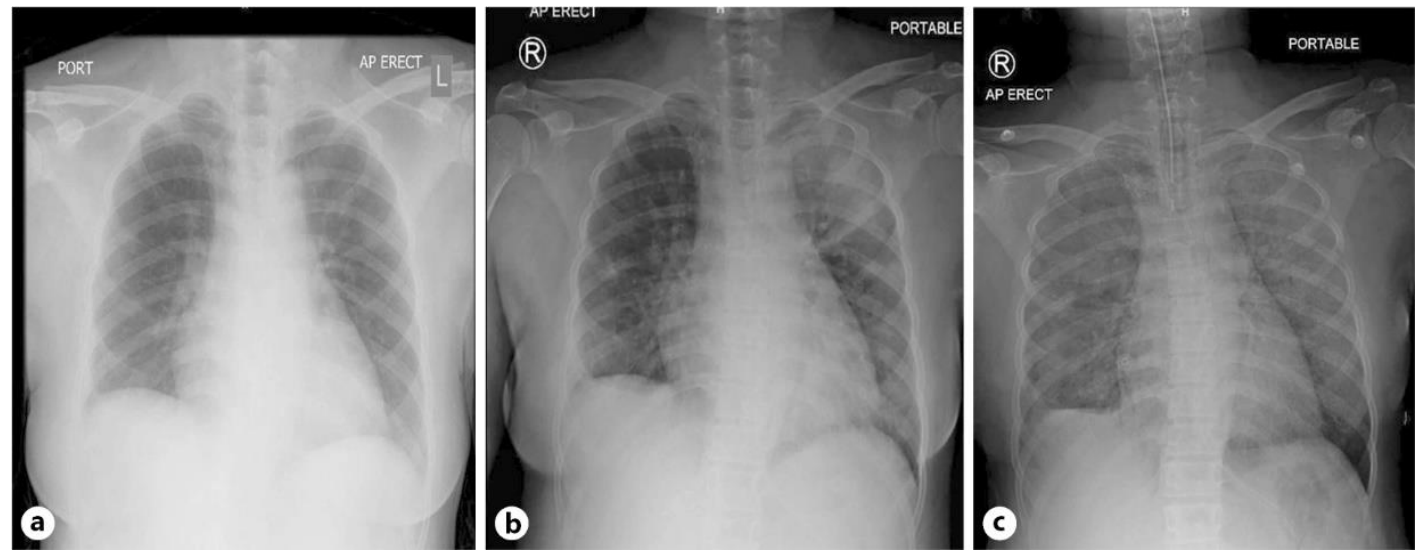

Fig. 1. Progressive CXR changes in COVID-19 disease. a Day 1 CXR: no significant radiological changes. b Day 4 CXR: new left upper zone consolidation. c Day 6 CXR: bilateral widespread pulmonary infiltrates. COVID-19, coronavirus disease 2019; CXR, chest X-ray.

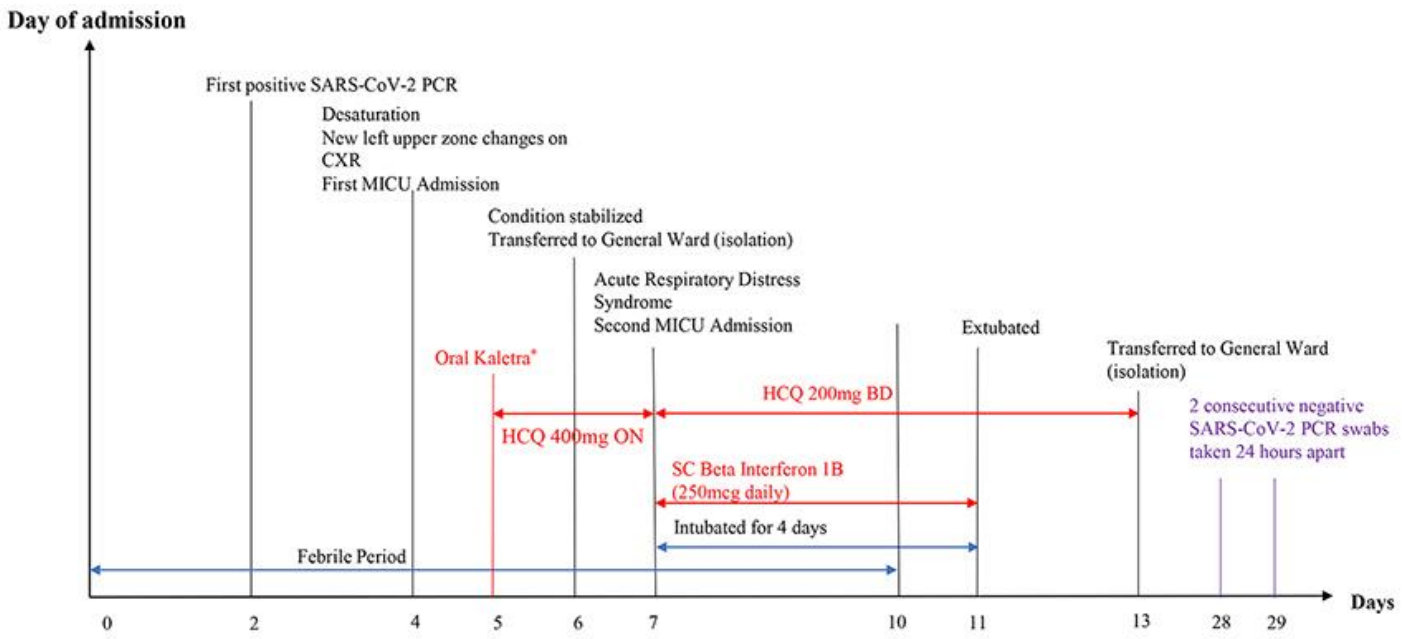

Fig. 2. Clinical course of COVID-19 in an ESKD patient. COVID-19, coronavirus disease 2019; CXR, chest X-ray; ESKD, end-stage kidney disease; HCQ, hydroxychloroquine; MICU, medical intensive care unit; PCR, polymerase chain reaction; $\mathrm{SC}$, subcutaneous. 


\section{Case Reports in Nephrology and Dialysis}

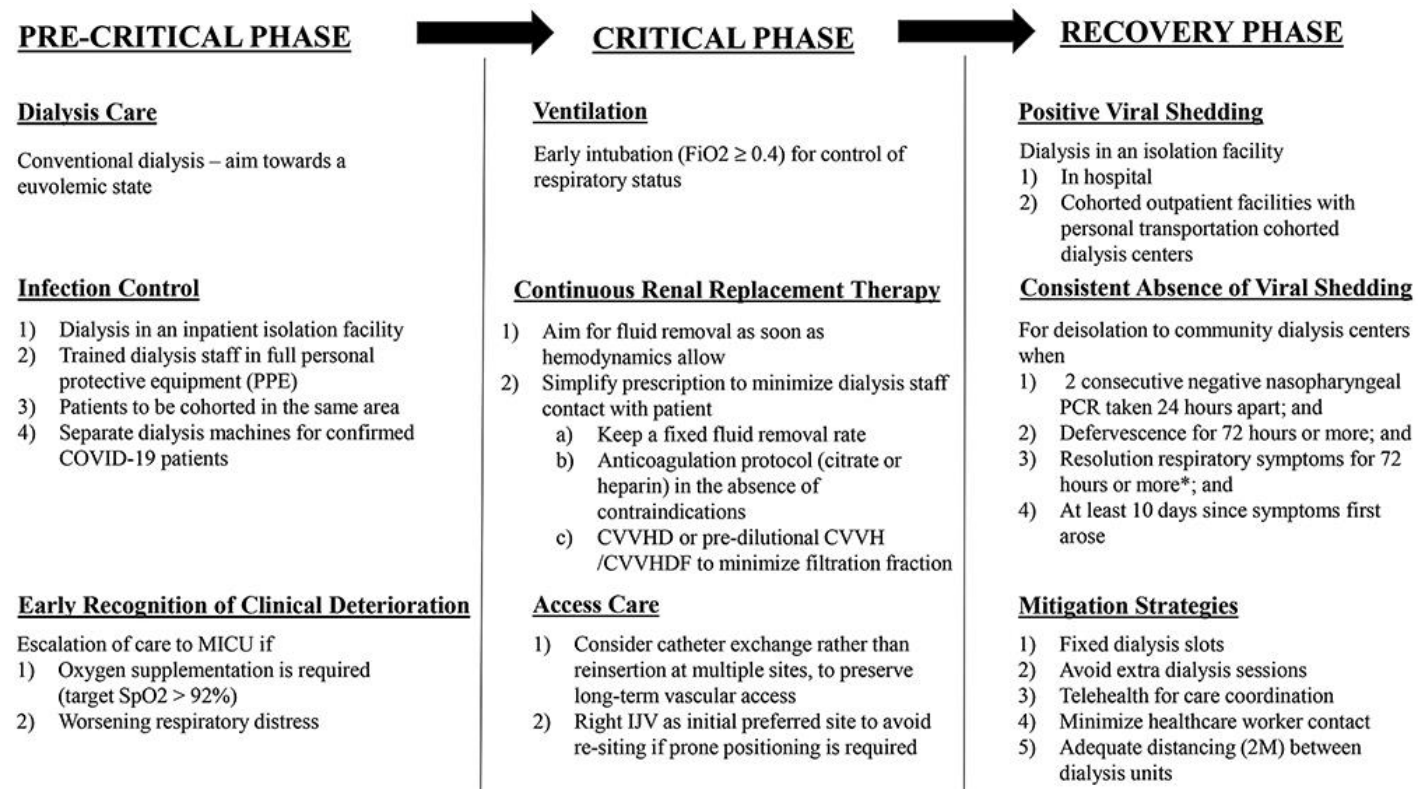

Fig. 3. Proposed workflow for the management of critical COVID-19 disease in ESKD patients. * Resolution of symptoms, e.g., absence of cough or shortness of breath. 2M, 2 meters; COVID-19, coronavirus disease 2019; CVVH, continuous venovenous hemofiltration; CVVHD, continuous venovenous hemodialysis; CVVHDF, continuous venovenous hemodiafiltration; ESKD, end-stage kidney disease; $\mathrm{FiO}_{2}$, fraction of inspired oxygen; IJV, internal jugular vein; MICU, medical intensive care unit; PCR, polymerase chain reaction; $\mathrm{SpO}_{2}$, peripheral oxygen saturation. 